\title{
Multi-transmission Lines Loaded by Linear and Nonlinear Lumped Elements: FDTD Approach
}

\author{
Ismail ALAOUI ABDALLAOUI ${ }^{1,2}$, Hassane KABBAJ ${ }^{1}$ and Philippe DESCAMPS ${ }^{2}$ \\ 1. Sidi Mohammed Ben Abdellah University-LSSC-B.P. 2202- Route d'Immouzzer, Fés, Morocoo \\ 2. Normandie University ENSICAEN CRISMAT UMR6580. 6 boulevard Maréchal Juin, 14050 Caen cedex 04, France
}

\begin{abstract}
In this paper, we are interested on studying the Multi-transmission Lines, the analysis is ensured by the FDTD (finite difference temporal domain) method combined with the ABC (absorbing boundary conditions) to predict the current and voltage behavior resulting from the electromagnetic fields all along lines. Simulated results will be compared to commercial software to validate the proposed algorithm.
\end{abstract}

Key words: Transmission lines, FDTD method, ABC-PML, Maxwell equations, lumped elements.

\section{Introduction}

The simplicity that offers the FDTD (finite difference temporal domain) method for solving Maxwell's differential equations makes this method suitable for analyzing electromagnetic problems. Moreover, it's easy to integrate the absorbing boundary conditions in the numerical computation domain. The stability and the precision is an important condition to design a time domain numerical algorithm.

Furthermore, the FDTD method allows to approach the nonlinear structures whose parameters vary according to time, although FDTD method solves the problem in time, simulates a bandwidth only with one simulation and provides frequency domain responses using the Fourier transform.

Simulation with the FDTD method takes an important time of calculation and an inordinate occupation of memory, especially, when a fine mesh is used on such 2.5 dimension. However, this method permits to know all values of electromagnetic fields at each point of space and time.

The organization of the paper is built around three

Corresponding author: Ismail ALAOUI ABDALLAOUI, Ph.D. student, member of LSSC \& LaMIPS laboratory, research fields: EMC \& antennas. E-mail: ismail.alaouiabdallaoui@usmba.ac.ma. sections: The first section deals with the presentation of the numerical method and its integration with $\mathrm{ABC}$ (absorbing boundary conditions). In the second section, design and modeling analysis of multi layers printed on laminate substrate and extending Maxwell's equation to integrate the lumped elements is presented. The correlation between full-wave time domain simulation FDTD and commercial software SPICE is discussed in third section.

\section{FDTD Method}

\subsection{Introduction}

The FDTD technique was first reported by Yee [1], this method is very useful to solve Maxwell's curl equations, passive, active and linear/nonlinear electromagnetics problems.

The differential time-domain Maxwell's curl equations over time in charge free regions containing materials without magnetic losses are:

$$
\begin{gathered}
\overrightarrow{R o t} \vec{E}=-\mu \frac{\partial \vec{H}}{\partial t}-\sigma_{m} \cdot \vec{H} \\
\overrightarrow{\operatorname{Rot}} \vec{H}=\varepsilon \frac{\partial \vec{E}}{\partial t}+\sigma_{e} \cdot \vec{E}
\end{gathered}
$$

where $\sigma_{e}, \sigma_{m}$ are electromagnetics losses, $\varepsilon$ and $\mu$ are respectively the permittivity and permeability of a 
dielectric medium [1-3]. Maxwell's Eqs. (3)-(8) can be written in Cartesian coordinates, as follow:

$$
\begin{array}{r}
\varepsilon \frac{\partial E_{x}}{\partial t}+\sigma_{e} \cdot E_{x}=\frac{\partial H_{z}}{\partial y}-\frac{\partial H_{y}}{\partial z} \\
\varepsilon \frac{\partial E_{y}}{\partial t}+\sigma_{e} \cdot E_{y}=\frac{\partial H_{x}}{\partial y}-\frac{\partial H_{z}}{\partial z} \\
\varepsilon \frac{\partial E_{z}}{\partial t}+\sigma_{e} \cdot E_{z}=\frac{\partial H_{y}}{\partial y}-\frac{\partial H_{x}}{\partial z} \\
-\mu \frac{\partial H_{x}}{\partial t}-\sigma_{m} \cdot H_{x}=\frac{\partial E_{z}}{\partial y}-\frac{\partial E_{y}}{\partial z} \\
-\mu \frac{\partial H_{y}}{\partial t}-\sigma_{m} \cdot H_{y}=\frac{\partial E_{x}}{\partial y}-\frac{\partial E_{z}}{\partial z} \\
-\mu \frac{\partial H_{z}}{\partial t}-\sigma_{m} \cdot H_{z}=\frac{\partial E_{y}}{\partial y}-\frac{\partial E_{x}}{\partial z}
\end{array}
$$

As we know, the FDTD algorithm solves the electromagnetic fields component at discrete points both in time and space. The period of time must be carefully chosen to guarantee the stability and convergence of the solution.

The choice of the period of sampling must comply with certain restrictions to guarantee the stability of the solution, it is demonstrated that the CFL (Courant-Fierdrichs-Levy) condition, ensures the stability of the FDTD algorithm, the relationship between increasing space and incrementing time is given [2-5]:

$$
\sqrt{\frac{1}{\Delta x^{2}}+\frac{1}{\Delta y^{2}}+\frac{1}{\Delta z^{2}}}>C v \cdot \Delta t
$$

where, $\Delta x, \Delta y$ and $\Delta z$ are the mesh grid, $C_{v}$ is the wave velocity in the dielectric substrate.

\subsection{Absorbing Boundary Conditions}

The size of the area that can be simulated using FDTD is limited by the machine resource. Indeed, no computer can storage unlimited amount of data. However, the FDTD problem space size must be finite to solve Maxwell's curl equations. The solution is to surround the system with the absorbing boundary conditions (ABC) [6-9].

Several various type of absorbing boundary conditions have been developed, but the most robust
ABC's is the PML (perfect matched layer) introduced by Berenger $[10,12]$, this approach is based on creating a nonphysical absorbing medium adjacent to the outer FDTD mesh boundary which is independent of the angles and frequencies of the wave incident on this boundary [4].

The main element of PML is the variation of conductivities based on parameters of PML, the current conductivity variation in the $\mathrm{z}$ direction is given by $\mathrm{Eq}$. (10).

$$
\left\{\begin{array}{c}
\sigma_{e, m}(z)=\frac{\sigma_{\text {Max }}\left|z-z_{0}\right|^{m}}{d^{m}} \sim \\
\sigma_{\text {Max }}=\frac{m+1}{150 \pi d z \sqrt{\varepsilon_{r}}} \stackrel{ }{*}
\end{array}\right.
$$

where, $\sigma_{\text {Max }}, m, d, \varepsilon_{r}$, represent the PML parameters, to known, electric/magnetic conductivity maximum, the polynomial degree, PML area thickness and dielectric constant respectively [13].

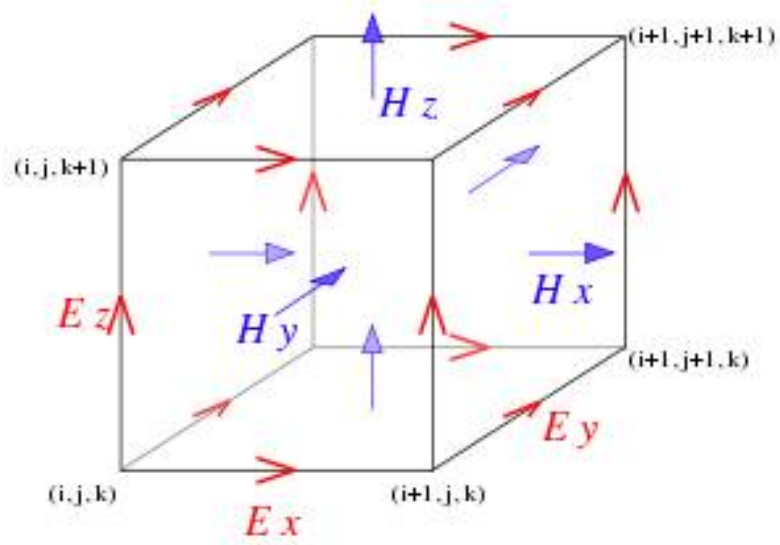

Fig. 1 Arrangement of field components on a Yee cell.

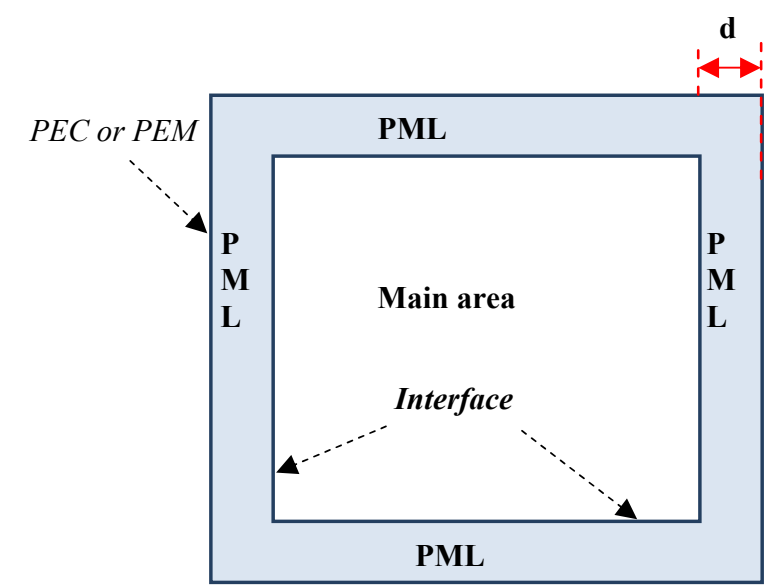

Fig. 2 PML layers surrounding the main area. 


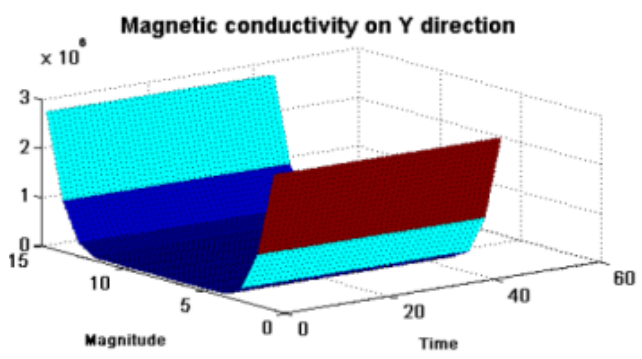

Electric conductivity on $\mathrm{X}$ direction

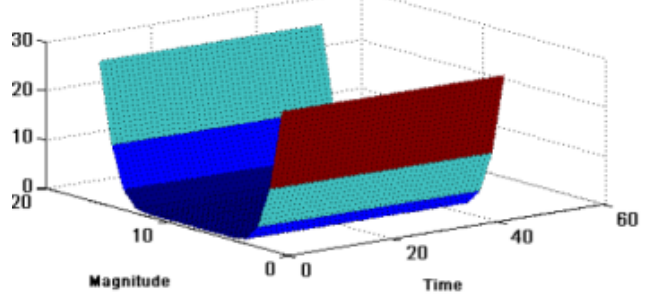

Fig. 3 Simulated PML layers.

\section{Multi-transmission Line}

\subsection{Design, Modeling and Analysis}

As mentioned above, this section will be dedicated to design the numerical computation domain and the components of circuit to predict the system behavior.

We assumed that we deal with three materials, free space, metal and FR4 dielectric.

The physical characteristics of the structure depend on its dimensions [13] which are summarized in Table 1.

(1) Modeling of lumped circuit elements

Modeling of the connection of linear and nonlinear lumped circuit elements to a micro strip trace will be discussed, because, many practical electromagnetics application require inclusion of lumped circuit elements [3, 13-15].

The electric current flowing through these circuit elements can be expressed by current density term, $J_{L}$ and can be accounted in Maxwell's curl equations [3, $4,16-18]$.

$$
\overrightarrow{R o t} \vec{H}=\varepsilon \frac{\partial \vec{E}}{\partial t}+\overrightarrow{J_{C}}+\overrightarrow{J_{L}}
$$

The relationship between the current density and the current of the lumped element localized at a unit cell $(i$, $j, k)$ is given by:
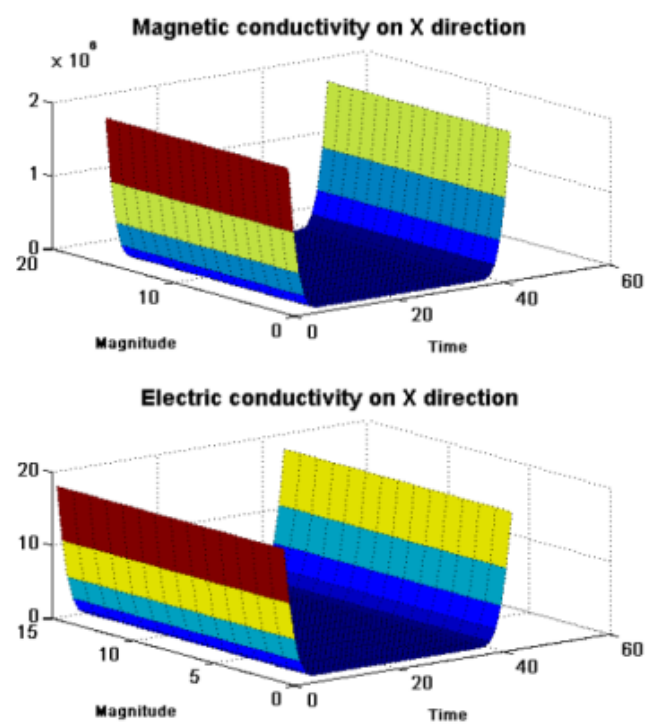

$$
J_{Z}^{n+\frac{1}{2}}(i, j, k)=\frac{I_{L}{ }^{n+\frac{1}{2}}(i, j, k)}{d x d y}
$$

(a) Insertion of linear lumped elements

For any electromagnetic simulation, it's important to include incident fields from far or near zones sources to excite the objects in a problem space.

To place a voltage source $V_{i n}$ with internal resistor $R_{i n}$, between two nodes and located at the field component $E_{z(i, j, k)}$, using the discrete domain [14, 16-19], the current density can be written as:

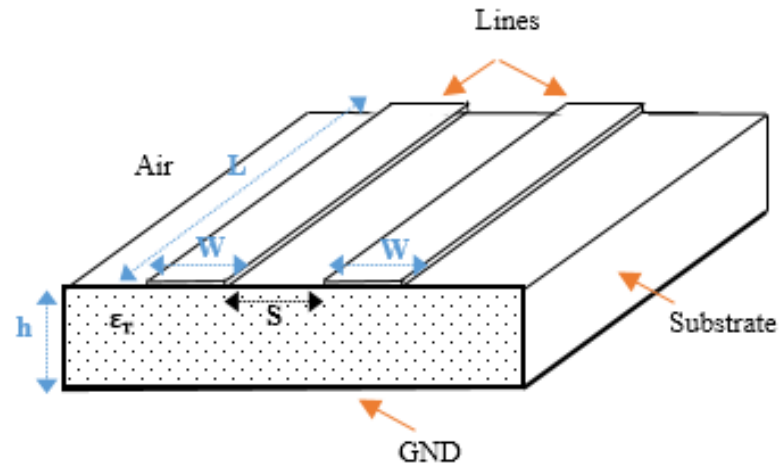

Fig. 4 The proposed microwaves circuit.

Table 1 Dimensions of the proposed structure.

\begin{tabular}{|l|l|l|l|l|}
\hline $\mathrm{L}(\mathrm{mm})$ & $\mathrm{H}(\mathrm{mm})$ & $\mathrm{W}(\mathrm{mm})$ & $\mathrm{S}(\mathrm{mm})$ & $\varepsilon_{\mathrm{r}}$ \\
\hline 70 & 4 & 6 & 10 & 4.8 \\
\hline$\Delta_{x}(\mathrm{~mm})$ & $\Delta_{\mathrm{y}}(\mathrm{mm})$ & $\Delta_{Z}(\mathrm{~mm})$ & $Z_{0}(\Omega)$ & $\mathrm{N}_{\mathrm{y}}(\mathrm{mm})$ \\
\hline 1 & 1 & 1 & 50 & 70 \\
\hline
\end{tabular}




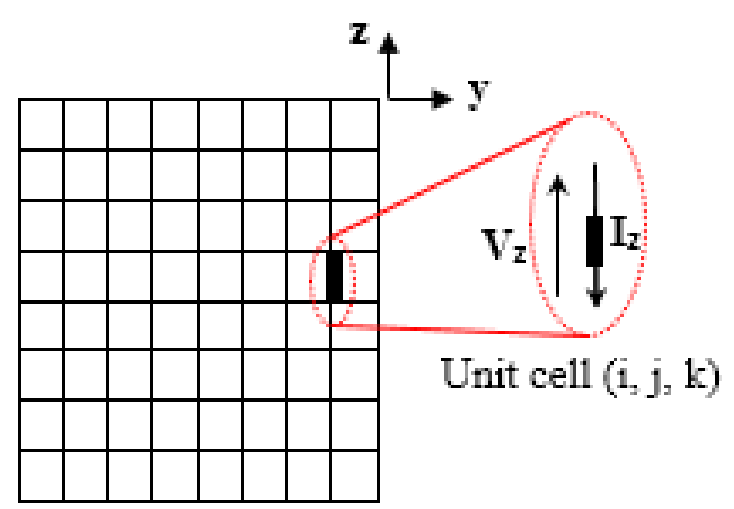

Fig. 5 Lumped element placed at unit cell $(i, j, k)$.

$$
J_{z}^{n+\frac{1}{2}}(i, j, k)=\frac{V_{i n}{ }^{n+\frac{1}{2}}}{R_{S}}=\frac{d z}{R_{S}}\left(\frac{E_{z}{ }^{n+1}(i, j, k)+E_{z}{ }^{n}(i, j, k)}{2}\right)+
$$

For inserting capacitor located at the field component $E_{z(i, j, k)}$ with $C$ farads capacitance, the current density can be written in discrete time and space as:

$$
\begin{aligned}
J_{z}{ }^{n+\frac{1}{2}}(i, j, k)= & \frac{C \times d z}{d t}\left(E_{z}{ }^{n+1}(i, j, k)\right. \\
& \left.-E_{z}{ }^{n}(i, j, k)\right)
\end{aligned}
$$

Eqs. (13) and (14) can be easily added to FDTD algorithm to calculate the electric field $E_{z}{ }^{n+1}(i, j, k)$ at location of the lumped elements.

(b) Insertion of nonlinear lumped elements

One of the most strengths of the FDTD method is to model nonlinear component, or the lumped element is characterized by nonlinear voltage-current relation in discrete time and space $[4,15,16,18]$.

For inserting a lumped circuit diode located at the field component $E_{z(i, j, k)}$ in the negative zdirection, the current is expressed by:

$$
I_{d}=I_{0}\left(e^{\left(\frac{q \cdot V_{d}}{N k t}\right)}-1\right)
$$

where $q$ is the absolute of electron charge, $k$ is Boltzmann's constant, and $T$ is the absolute temperature in Kelvins. The current equation can be written in discrete form as:

$$
J_{z}^{n+\frac{1}{2}}(i, j, k)=
$$

$\frac{I_{0}}{d x \times d y}\left[e^{\left(\frac{q . d z}{2 . k . T}\right)\left(E_{z}{ }^{n+1}(i, j, k)+E_{z}{ }^{n}(i, j, k)\right)}-1\right]$

To solve numerically the nonlinear equation located at the field component $E_{z(i, j, k)}$, it is necessary to use the Newton-Raphson method $[4,14]$.

\section{Results and Discussion}

The proposed circuit, is composed of two coupled lines, the active line is loaded by nonlinear lumped element, the passive line is loaded by linear lumped capacitor $C=4 p F$.

The used excitation is a matched resistive voltage source $\left(R_{i n}=Z_{0}=50 \Omega\right)$ providing a $5 \mathrm{~V}, 2 \mathrm{GHz}$ as sinusoidal signal.

$V_{S 1}, V_{S 2}$ are the voltage across the numerical lumped elements terminating a strip line, diode and capacitor respectively.

The electromagnetic energy from $V_{\text {in }}$ travels along the active transmission line. The coupled electromagnetic energy is ensured via electromagnetic field coupling and appears as a voltage across the source - near crosstalk - and load - far crosstalk-of the passive transmission line. The FDTD method simulates electric and magnetic fields throughout the line, and can easily deduce the voltage and current on the source and load ends for both lines [3, 4, 14, 18$]$.

To validate the FDTD algorithm, we compare the results with the commercial software SPICE.

The two transmission lines are coupled, and the coupling capacitance is order of $C_{0}=3.21687 \mathrm{e}-11 \mathrm{~F} / \mathrm{m}$.

The results of the proposed circuit, show a good correlation between the commercial software SPICE

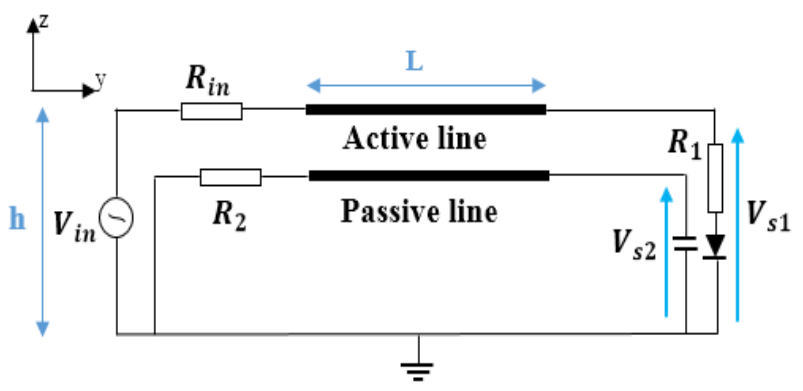

Fig. 6 Multilayers circuit loaded by the lumped elements. 

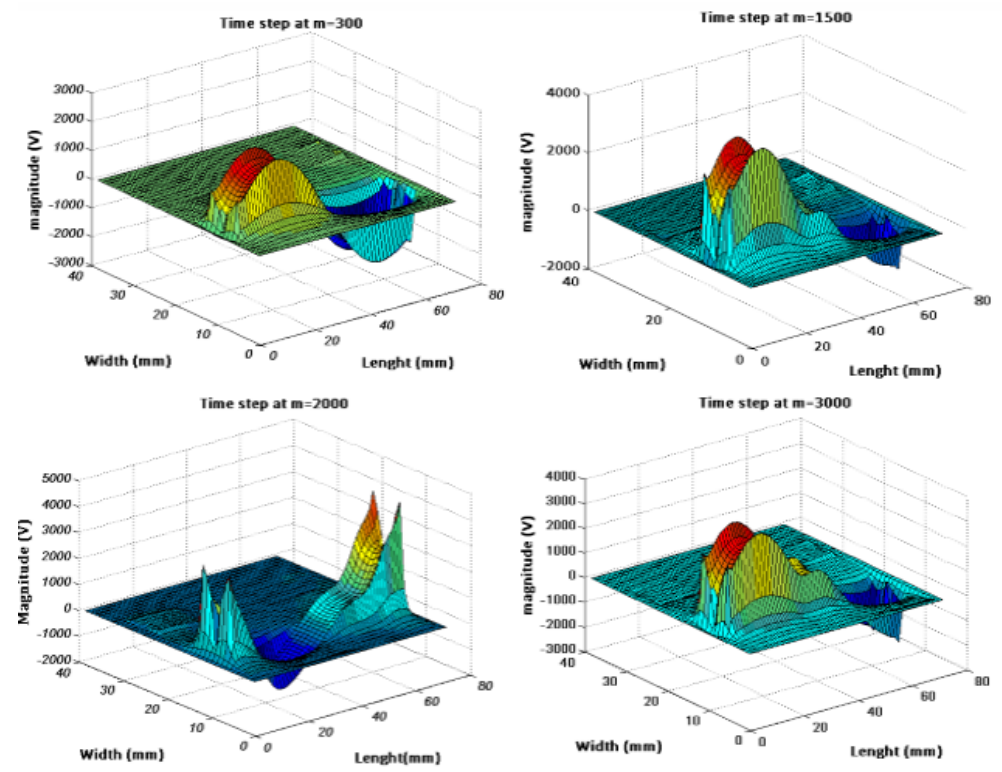

Fig. 7 Ez electric field propagation in space at $t=300, t=1,500, t=2,000$ and $t=3,000$.

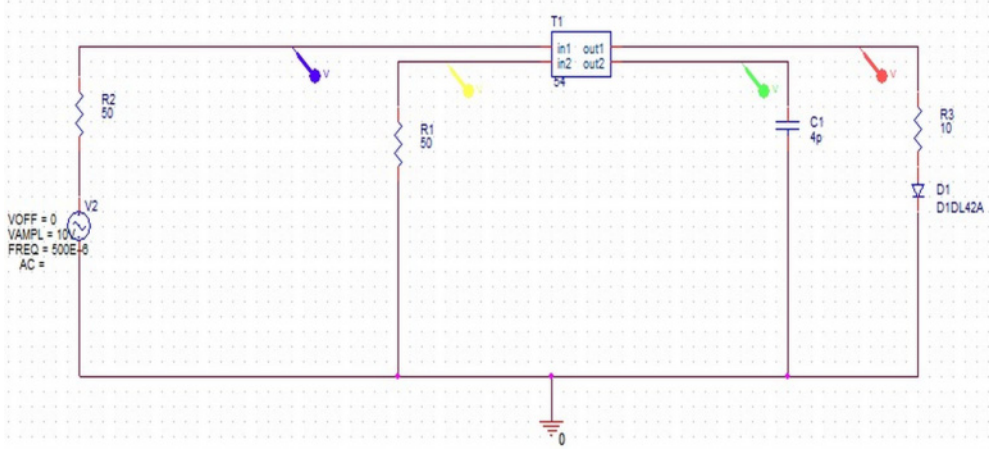

Fig. 8 The design circuiton SPICE.

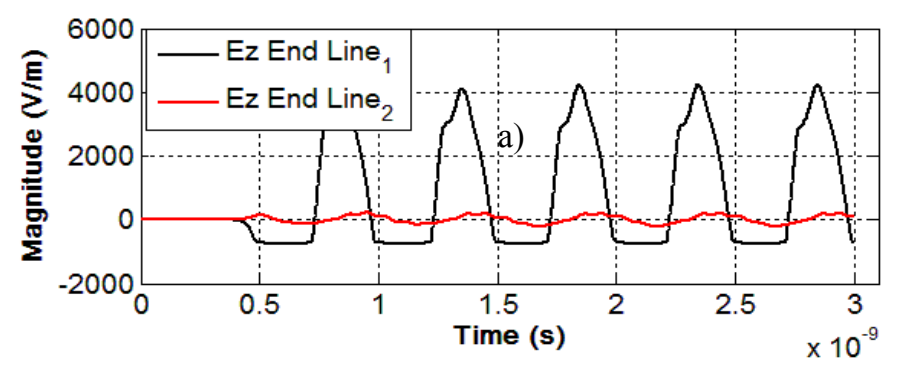

(a)

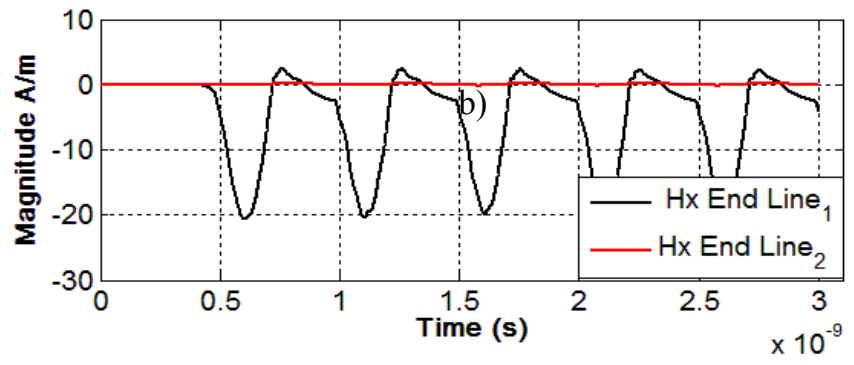

(b)

Fig. 9 (a) Electric and (b) magnetic field obtained by FDTD algorithm at the load ends of passive and active line. 


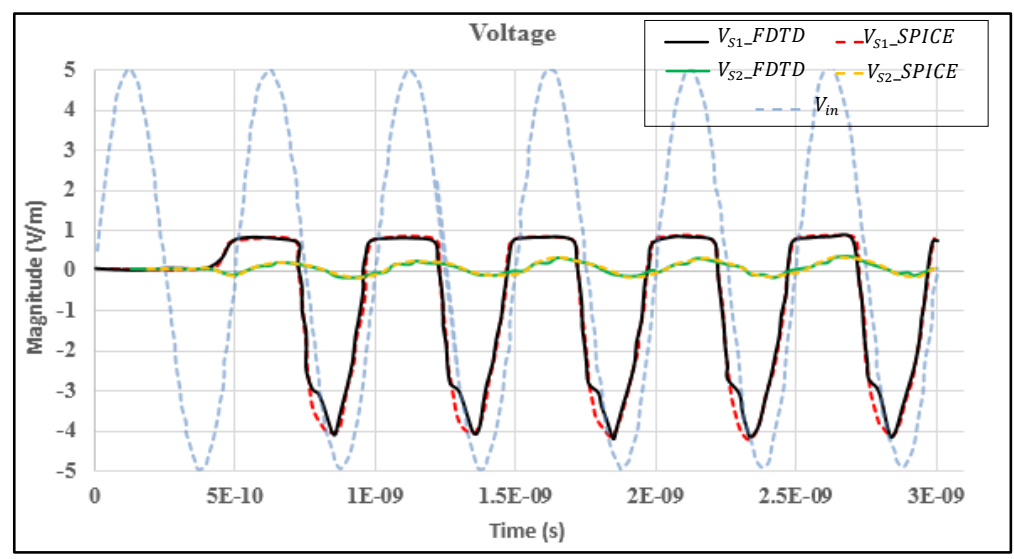

Fig. 10 Voltage at the load ends of passive and active line.

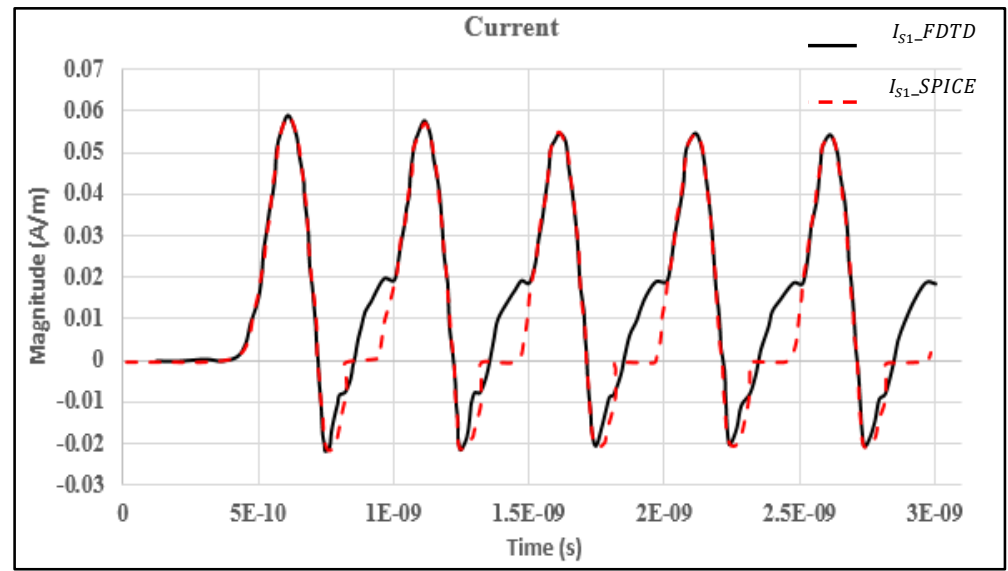

Fig. 11 Current at the load ends of passive and active line.

and the proposed algorithm based on FDTD time domain integrated with the ABC's.

The observed difference is due to establishment and size of the mesh and the rounding of numbers.

\section{Conclusion}

In this paper, we have developed an algorithm 2.5D full wave time domain based on FDTD method to solve Maxwell's curl equations, including the absorbing boundary conditions and arbitrary lumped elements.

The modeling results of the proposed structure including non-linearity are in agreement with the commercial software SPICE and confirm the validity of the proposed algorithm.

The FDTD-PML method, it's able to be developed for several electromagnetics applications such as: non uniform and multi-transmissions lines loaded by the complexes and nonlinear lumped elements transistors (MOSFET and MESFET), the patch antenna, the couplers and filters.

\section{References}

[1] Yee, K. S. 1966. "Numerical Solution of Initial Boundary Value Problems Involving Maxwell's Equations in Isotropic Media." IEEE Trans. Antennas Propagation. AP-14: 302-7.

[2] Alejandro Duenas, J. 2D electromagnetic Simulation of Passive Microstrip Circuits. International Standard Book Number 13: 978-1-4200-8705-5.

[3] Taflove, A. 1995. Computational Electrodynamics. Finit Difference Temporal Domaine Technique. Boston, 1995, British library, artech house, INC.

[4] Elsherbeni, A., and Demir, V. 2009. The Finite-Difference Time-Domain Method for Electromagnetics with Matlab Simulations. The Institution of Engineering and 
Technology, USA.

[5] Sullivan, D. M. 2000. Electromagnetic Simulation Using the FDTD Method. New York: IEEE Press on RF and MICROWAVE TECHNOLOGY.

[6] Bayliss and Turkel Radiation Boundary Condition for Wave-Like Equation. ICASE Report No. 79-26, October 1979.

[7] Mur, G. 1981. "Absorbing Boundary Conditions for the Finit Difference Approximation of the Time Domain Electromagnetic Field Equations." IEEE trans. Electromagn. Compat 23: 377-82.

[8] Engquist, B., and Majda, A. 1977. "Absorbing Boundary Conditions for the Numerical Simulation of Waves." Mathematics of Computation 31: 629-51.

[9] Halpern, L., and Trefethen, L. N. 1986. "Wide-Angle One-Way Wave Equations." Numerical Analysis Report 86-5, Dept. of Mathematics, Massachusetts Institute of Technology.

[10] Berenger, J.-P. 1994. "A Perfectly Matched Layer for the Absorption of Electromagnetic Waves." Journal of Computational Physics 114: 185-200.

[11] Katz, D. S., Thiele, E. T., and Taflove, A. 1994. "Validation and Extension to Three Dimensions of the Berenger PML Absorbing Boundary Condition for FD-TD Meshes." Microwave Guided Wave lett., 268-70.

[12] Berenger, J.-P. 1994. “A Perfectly Matched Layer for Free
Space Simulation in FDTD Codes." Presentation at EUROEM'94, Bordeaux, France, May.

[13] Gedney, S. D. 1996. “An Anisotropic Perfectly Matched Layer Absorbing Media for the Truncation of FDTD Lattices." IEEE Transactions on Antennas Propagation 12: 1630-9.

[14] Clayton, P. 2006. Electromagnetic Compatibility. 2nd ed. New Jersey: John Wiley \& Sons, Inc.

[15] Thesis of Labiod Samir.. "Contribution à l'étude de perturbations électromagnétiques sur des composants MOS en Utilisant la méthode FDTD.” Algeria, 2013.

[16] Ciampolini, P., Mezzanotte, P., Roselli, L., and Sonentino, R. 1996. "Accurate and Efficient Circuit Simulation with Lumped-Element FDTD Technique." Transactions on Microwave Theory and Techniques 44 (12): 2207-15.

[17] Kalialakis, C. 1999. Finite Difference Time Domain Analysis of Microstrip Antenna-Circuit Models. School of Electronic and Electrical Engineering, Bermingham.

[18] May, M. P., Taflove, A., and Baron, J. 1994. "FD-TD Modeling of Digital Signal Propagation in 3-D Circuit with Passive and Active Loads." IEEE Transaction on Microwave Theory and Techniques 42 (8).

[19] Sui, W. Q., Christensen, D. A., and Durney, C. H. 1992. "Extending the Two Dimensional FDTD Method to Hybrid Electromagnetic Systems with Active and Passive Lamped Elements." IEEE Transaction on Microwave Theory. 